\title{
Erratum
}

\section{The Rotation Number for Almost Periodic Potentials}

\author{
R. Johnson ${ }^{1}$ and J. Moser ${ }^{2}$ \\ 1 Department of Mathematics, University of Southern California, Los Angeles, CA 90007, USA \\ 2 ETH-Zentrum, CH-8092, Zürich, Switzerland
}

Commun. Math. Phys. 84, 403-438 (1982)

It has been pointed out to us by B. Simon that the proof of Proposition 3.3, p. 413, is incorrect. The statement, however, is valid as it stands and we want to present a proof of that statement:

According to Proposition 2.1, we have to show that $m_{+}\left(x+t_{n}\right)$ converges uniformly for any sequence $t_{n}$ for which $q\left(x+t_{n}\right)$ does. We show first the convergence for $x=0$ and subsequently verify the uniform convergence for all $x \in \mathbb{R}$

We change the notation and write for fixed $z$ in $\operatorname{Im} z \neq 0$,

$$
m[q]=m_{+}(0, z ; q) .
$$

Proposition. If $q_{n}, q$ are bounded continuous functions and $q_{n} \rightarrow q$ uniformly on compact sets, then $m\left[q_{n}\right] \rightarrow m[q]$.

The proof can be based on $\mathrm{H}$. Weyl's construction of the limit point $m[q]$. (See, e.g., in [6, Chap. IX, Sect. 2] of our paper.) If $\varphi=\varphi_{b}(x, z, \theta)$ is a nontrivial solution of $(L-z) \varphi=0$ satisfying $\varphi^{\prime} \cos \theta+\varphi \sin \theta=0$ for $x=b$, then

$$
C_{b}=\left\{\left.\frac{\varphi^{\prime}}{\varphi}\right|_{x=0}, 0 \leqq \theta \leqq 2 \pi\right\}
$$

defines a circle in the complex plane which for $b \rightarrow \infty$ shrinks to the limit point $m[q]$. Denoting by $D_{b}$ the closed disc bounded by $C_{b}$, one has $D_{b^{\prime}} \subset D_{b}$ for $b^{\prime}>b$ (strict containment). In particular, $m[q]$ is contained in all $D_{b}$.

If now $D_{b}^{n}$ denote the corresponding discs for $q_{n}$, we can, for $\varepsilon>0$, choose $b$ so large that the radius of $D_{b}$ is $<\varepsilon / 2$. Fix $b^{\prime}>b$ and note that clearly $C_{b^{\prime}}^{n} \rightarrow C_{b^{\prime}}$ as $n \rightarrow \infty$, i.e. for $n>N$ we have

$$
m\left[q_{n}\right] \in D_{b^{\prime}}^{n} \subset D_{b} .
$$

Hence both $m\left[q_{n}\right]$ and $m[q]$ are contained in $D_{b}$, i.e. satisfy $\left|m\left[q_{n}\right]-m[q]\right|<\varepsilon$ for $n>N$, proving the proposition.

We apply this proposition to the translates $q_{t}=q(x+t)$ of $q \in \mathscr{A}(\mathscr{M})$, and observe that

$$
m\left[q_{t}\right]=m_{+}(t, z), \quad \operatorname{Im} z \neq 0 .
$$


Thus if $\left\|q_{t_{n}}-q^{*}\right\|_{\infty} \rightarrow 0$, we have $m\left[q_{t_{n}}\right] \rightarrow m\left[q^{*}\right]$, and it follows that $m[\cdot]$ can be extended to a continuous functional on the hull $E(q)$, the closure of $\left\{q_{t}\right\}$. Since $E(q)$ is compact, $m[\cdot]$ is actually uniformly continuous and therefore

$$
m\left[q_{t+t_{n}}\right]-m\left[q_{t}^{*}\right]=m\left(t+t_{n}, z\right)-m^{*}(t, z)
$$

tends to zero uniformly in $t \in \mathbb{R}$, since

$$
\left\|q_{t+t_{n}}-q_{t}^{*}\right\|_{\infty}=\left\|q_{t_{n}}-q^{*}\right\|_{\infty} \rightarrow 0 .
$$

There are a number of different ways to prove Proposition 3.3, for example, by using Scharf's argument, [23]. Also B. Simon suggested another simple proof and we thank him for this communication as well as for pointing out our mistake. Finally we note that the above proposition is stronger than necessary since it does not require uniform convergence of $q_{n} \rightarrow q$ on the whole axis.

Communicated by A. Jaffe

Received May 5, 1983 